\title{
Constructive theory of Banach algebras
}

\author{
THIERRY COQUAND \\ BAS SPITTERS
}

\begin{abstract}
We present a way to organize a constructive development of the theory of Banach algebras, inspired by works of Cohen, de Bruijn and Bishop [15, 14, 4, 19]. We illustrate this by giving elementary proofs of Wiener's result on the inverse of Fourier series and Wiener's Tauberian Theorem. In a sequel to this paper we show how this can be used in a localic, or point-free, description of the spectrum of a Banach algebra.
\end{abstract}

2000 Mathematics Subject Classification 46Jxx,06D22,03F60 (primary)

Keywords: Banach algebra, constructive mathematics, Gelfand-Mazur theorem, Wiener Tauberian theorem

\section{Introduction}

The applications of the theory of Banach algebras to 'concrete' theorems in analysis, such as Wiener's theorem on the inverse of Fourier series, or Wiener's Tauberian Theorem [31], constitute a striking example of the power of abstract methods in mathematics. The abstract argument is short and easy to grasp, when compared to Wiener's explicit constructions. Furthermore, it is highly non-constructive and uses Zorn's Lemma. As such, it is a perfect illustration of Hilbert's defense of the use of the law of excluded-middle against Brouwer [20]. A natural question is whether the abstract argument does not contain, in some implicit way, an actual construction. This question has been analysed and answered by P. Cohen [15]. Closely related are later works by de Bruijn and van der Meiden [14], and by Bishop and Bridges [4]. Applications of the latter may be found in $[6,7,10,11,8,12,9,29]$. Here we present a slightly different analysis of the non-constructive argument, thus providing an elementary treatment. Furthermore it allows us, in a sequel to the present paper, to reformulate some key results of Bishop and Bridges [4] in a localic, or point-free, setting. This will generalize previous localic treatments of Gelfand theory for $\mathrm{C}^{*}$ algebras $[2,1,18]$. 
This paper is organised as follows. The first section explains informally the main idea of the paper. The next section is a detailed presentation of the elementary theory of Banach algebras. Hopefully it can be readily implemented in type theory using the framework presented in [27]. This presentation follows ideas presented in [5]. The remaining sections provide our main applications.

\section{Notation}

We use the following conventions, unless otherwise indicated:

$A, B$ for Banach algebras.

$a, b, c, \ldots$ for elements of a Banach algebra.

$\varphi, \psi$ for functionals.

$\Gamma_{R}$ denotes the circle with radius $R, \Gamma$ is the circle with radius 1 .

\section{Analysis of the abstract reasoning}

Let $A$ be a commutative Banach algebra with unit, and let MFn $A$ be the compact space of non-zero multiplicative functionals $A \rightarrow \mathbb{C}$ with the weak-* topology. Let us assume that $f$ in $A$ satisfies $\varphi(f) \neq 0$ for all $\varphi$ in $\operatorname{MFn} A$. We want to show that $f$ is invertible in $A$. To obtain a contradiction, we assume that $f$ is not invertible. Then the ideal $\langle f\rangle$ is proper, and hence, using Zorn's Lemma, included in a maximal ideal $\mathfrak{m}$. This maximal ideal is necessarily closed. Using the Gelfand-Mazur theorem, the algebra $A / \mathfrak{m}$ is isomorphic to $\mathbb{C}$. The corresponding multiplicative functional $\varphi_{\mathfrak{m}}: A \rightarrow A / \mathfrak{m}$ is such that $\varphi_{\mathfrak{m}}(f)=0$. This contradicts the hypothesis on $f$. For example, the application of this argument to $A=l^{1}(\mathbb{Z})$ gives Wiener's theorem on inverse of Fourier series.

Cohen [15] observes that, instead of working with a maximal ideal, one can work just as well with the closure $I$ of the ideal generated by $f$. Since $x$ is invertible if $|1-x|<1$, an ideal contains 1 if and only if its closure contains 1 . Finally, the proof of the Gelfand-Mazur Theorem actually gives a more general result: if $A$ is a non-trivial Banach algebra, the spectrum of any element $u$ in $A$ is non-empty. A combination of these remarks eliminates the use of Zorn's Lemma.

To simplify, we consider the case where $A$ is generated by one element $u$ : for every $a$ in $A$ and $r>0$ there is a polynomial $P$ such that $|a-P(u)|<r$. The disc algebra [15] 
is such an algebra. In this case the spectrum MFn $A$ can be identified with the spectrum, $\sigma(u)$, of $u$ : that is, the set of complex $\lambda$ such that $\lambda-u$ is not invertible. Any such element $\lambda$ defines a multiplicative functional $\varphi(P(u)):=P(\lambda)$ on polynomials in $u$. If $|P(\lambda)|>|P(u)|$, then $P(\lambda)-P(u)$ is invertible, and hence $\lambda-u$ is invertible. Since this is not the case, $|P(\lambda)| \leqslant|P(u)|$, so $\varphi$ can be extended to a multiplicative functional $\varphi(g):=g(\lambda)$. Conversely, for $\varphi$ in $\operatorname{MFn} A$,

$$
\varphi(\varphi(u)-u)=\varphi(u)-\varphi(u)=0 .
$$

Hence $\varphi(u)-u$ cannot be invertible, that is $\varphi(u) \in \sigma(u)$. The hypothesis on $f$ becomes $f(\lambda) \neq 0$ for all $\lambda$ in the spectrum of $u$. The spectrum of $u$ is empty in $A / I$. Indeed, the closure of the ideal generated by $\lambda-u$ contains $f(\lambda)-f=f(\lambda)(\bmod I)$ which is invertible, and so $\lambda-u$ is always invertible $(\bmod I)$. Consequently, 1 belongs to $I$, and hence $f$ is invertible. The maximal ideal of the abstract argument has been replaced by the 'big enough' ideal $I$. Essentially, this is the method followed by de Bruijn and van der Meiden [14], who advocated a point-free approach to the description of the spectrum of a Banach algebra.

In this way we eliminate the use of Zorn's Lemma, but the argument is still nonconstructive. Cohen shows that we can follow the proof of Gelfand-Mazur and produce an actual computation of the inverse of $f$. Bishop and Bridges' work is similar [4]. Coquand and Stolzenberg [19] show that in most cases, we can obtain a relatively short and explicit formula for the inverse.

In this paper we suggest a slightly different constructive argument. It is enough to have a constructive proof of the following result.

Theorem 1 Let $u$ in $A$ be such that for all $\lambda, \lambda-u$ is invertible, and the inverse is uniformly bounded. Then $1=0$ in $A$.

Constructively, we have to state explicitly that the inverse is uniformly bounded. At first the conclusion seems purely negative, since it says that a ring is trivial. But if we apply the Theorem to a quotient algebra $A / I$, the conclusion shows that 1 is in $I$. Choosing $I$ to be the closure of $\langle f\rangle$ actually builds an inverse of $f$, provided we work constructively. Such use of 'trivial rings' occurs often in constructive algebra $[28,24]$. In the applications to Wiener's Theorems on Fourier series and to the Tauberian Theorem, we believe that our treatment is simpler than the one in [4]. Moreover, this theorem has a rather direct proof; for instance, it does not rely on Cauchy's formula like the treatment in $[15,4,19]$.

This constructive reading does not yet have the 'elegance' of the classical proofs, since we have to deal explicitly with a bound of the inverse. In the sequel to this paper, we 
explain how to 'hide' explicit mentions to this bound, by using a localic, or pointfree, presentation of the spectrum. In this way we obtain a rather faithful constructive explanation of the classical arguments.

\section{Preliminaries}

Most of the material in this section is well-known. We present it here to emphasize its elementary nature.

\subsection{Integration and differentiation with values in a Banach space}

We consider a vector space $E$ over the real numbers with an upper real valued seminorm: that is, for $a$ in $E,|a|$ is an upper real (open non-empty upper set of rationals) such that $|r a|=|r||a|$ and $|a+b| \leqslant|a|+|b|$. This is an important difference from Bishop's treatment, for whom the norm is a Cauchy real. ${ }^{1}$ The equality in $E$ is such that $|a|=0$ if and only if $a=0$ in $E$.

Let $f:[a, b] \rightarrow E$ be a (uniformly) continuous map. We say that $f$ is differentiable if and only if there exists a continuous $f^{\prime}:[a, b] \rightarrow E$ such that for all $\varepsilon>0$ there exists $\eta$ such that

$$
\left|f(y)-f(x)-(y-x) f^{\prime}(x)\right| \leqslant \varepsilon|y-x| \quad \text { if }|y-x| \leqslant \eta .
$$

Lemma 1 If $\left|f^{\prime}\right| \leqslant M$ on $[a, b]$, then $|f(b)-f(a)| \leqslant M(b-a)$. In particular, if $f^{\prime}=0$ on $[a, b]$, then $f(b)=f(a)$.

Proof For $\varepsilon>0$ we show $|f(b)-f(a)| \leqslant(M+\varepsilon)(b-a)$, by cutting $[a, b]$ in subintervals $\left[a_{i}, a_{i+1}\right]$ such that $\left|f\left(a_{i+1}\right)-f\left(a_{i}\right)-\left(a_{i+1}-a_{i}\right) f^{\prime}\left(a_{i}\right)\right| \leqslant \varepsilon\left(a_{i+1}-a_{i}\right)$.

\footnotetext{
${ }^{1}$ The fact that the norm is not a Cauchy real arises constructively if one wants to quotient a Banach space by a closed subspace which may not be located. This will indeed occur in the applications.

Another motivation is the interpretation of these results in Banach algebra bundles which we will discuss in Section 6. In a Banach algebra bundle the function $x \mapsto\left|a_{x}\right|$ is upper semi continuous, but generally not continuous; see [22].

Bishop and Bridges [4, p.462] state: 'It would be interesting, and probably non-trivial, to extend the theory to cover such algebras [where the norm is not a Cauchy real].'
} 
A normed vector space is complete if every Cauchy approximation converges. A Banach space is a complete normed vector space. Let $E$ be a Banach space. For uniformly continuous $g:[a, b] \rightarrow E$, we can define the integral $\int_{a}^{b} g$. We derive the usual properties. For instance, if $|g| \leqslant M$ on $[a, b]$, then $\left|\int_{a}^{b} g\right| \leqslant M(b-a)$.

Let $G(y):=\int_{a}^{y} g$. Then $G:[a, b] \rightarrow E$ is differentiable, and $G^{\prime}=g$.

\subsection{Exponential function}

A (commutative) Banach algebra $A$ is a Banach space with a ring structure, multiplication $a b$ being such that $|a b| \leqslant|a||b|$. In this subsection, we assume moreover that $A$ has a unit element 1 .

Lemma 2 If $|1-x|<1$, then $x$ is invertible with inverse $\sum(1-x)^{n}$.

Corollary 1 Let $I$ be an ideal. If $1 \in \bar{I}$, then $1 \in I$.

Proof By the previous lemma $I$ contains an invertible element.

We define the exponential of $a$ in $A$.

$$
e^{a}:=\sum \frac{a^{n}}{n !} .
$$

The map $x \mapsto e^{a x}$ defines a function $e_{a}: \mathbb{R} \rightarrow A$ which is differentiable, $e_{a}^{\prime}=a e_{a}$ and $e_{a}(0)=1$. Furthermore, $e_{a}(x+y)=e_{a}(x) e_{a}(y)$. In fact, these properties define the exponential function.

Lemma 3 Let $f: \mathbb{R} \rightarrow A$ be a continuous function such that $f(0)=1$ and $f(x+y)=$ $f(x) f(y)$. Then $f$ is differentiable, and $f=e_{a}$ with $a=f^{\prime}(0)$.

Proof We have $\frac{1}{t} \int_{0}^{t} f \rightarrow 1$ for $t \rightarrow 0$. So we can find $t>0$ such that $\left|1-\frac{1}{t} \int_{0}^{t} f\right|<1$. By Lemma 2, $v:=\frac{1}{t} \int_{0}^{t} f$ is invertible. Since $f(x+y)=f(x) f(y)$, we obtain

$$
t f(x) v=\int_{0}^{t} f(x+z) d z=\int_{x}^{x+t} f .
$$

Hence

$$
t f(x)=v^{-1} \int_{x}^{x+t} f=v^{-1}\left(\int_{0}^{t+x} f-\int_{0}^{x} f\right) .
$$

Journal of Logic \& Analysis 2:11 (2010) 
It follows that $f$ is differentiable and

$$
t f^{\prime}(x)=v^{-1}(f(x+t)-f(x)) .
$$

Since $f(x+y)=f(x) f(y)$, we have $f^{\prime}(x+y)=f(x) f^{\prime}(y)$. In particular, writing $a:=f^{\prime}(0)$, we have $f^{\prime}=a f$. Defining $g$ by $x \mapsto f(x) e_{a}(-x)$, we have $g(0)=1$ and

$$
g^{\prime}(x)=a f(x) e_{a}(-x)-a f(x) e_{a}(-x)=0 .
$$

By Lemma 1, we have $g=1$, and so $f=e_{a}$.

\subsection{Path integration}

Let $E$ be a Banach space over of the complex numbers. We say that $f: \mathbb{C} \rightarrow E$ is differentiable if and only if on each disc $\Delta_{r}$ there exists a (uniformly) continuous function $f^{\prime}: \Delta_{r} \rightarrow E$ such that for all $\varepsilon>0$ there exists $\eta>0$ such that for $\left|z^{\prime}-z\right| \leqslant \eta$

$$
\left|f\left(z^{\prime}\right)-f(z)-\left(z^{\prime}-z\right) f^{\prime}(z)\right| \leqslant \varepsilon\left|z^{\prime}-z\right|
$$

If $\gamma:[0,1] \rightarrow E$ is a differentiable function and $f: \mathbb{C} \rightarrow E$ is a continuous function, we define $\int_{\gamma} f:=\int_{0}^{1} f(\gamma(t)) \gamma^{\prime}(t) d t$. We say that $\gamma$ is a loop if and only if $\gamma(0)=\gamma(1)$.

Lemma 4 If $f: \mathbb{C} \rightarrow E$ is differentiable and $\gamma:[0,1] \rightarrow \mathbb{C}$ is a loop, then $\int_{\gamma} f=0$.

Proof We consider $g:[0,1] \rightarrow E$ defined by

$$
g(s):=\int_{0}^{1} f(s \gamma(t)) s \gamma^{\prime}(t) d t .
$$

Then $g(1)=\int f, g(0)=0$, and $g$ is differentiable. Moreover,

$$
g^{\prime}(s)=\int_{0}^{1}\left(f(s \gamma(t))+s \gamma(t) f^{\prime}(s \gamma(t))\right) \gamma^{\prime}(t) d t=\int_{0}^{1} h^{\prime}=h(1)-h(0)=0,
$$

where $h(t):=\gamma(t) f(s \gamma(t))$. By Lemma 1, $g(1)=g(0)=0$. Hence the result.

\section{Inverse function}

Let $A$ be a unital Banach algebra over the complex numbers.

Lemma 5 Let $a$ be invertible, with inverse $b$ bounded by $M$. Let $c<1$ and $|u| \leqslant \frac{c}{M}$. Then $a-u$ is invertible. Its inverse is bounded by $\frac{M}{1-c}$. 
Proof The element $a-u$ is invertible if and only if $1-u b$ is. Since $|u|<\frac{c}{M}$ and $|b|<M$, we have $|u b| \leqslant c$. Hence $1-u b$ is invertible by Lemma 2. The inverse is bounded by $\frac{1}{1-c}$. The inverse of $a-u$ is bounded by $\frac{M}{1-c}$.

We conclude that the set of invertible elements is open.

Theorem 2 Let $u$ be in $A$. If for all $z$ in $\mathbb{C}, z-u$ is invertible, with inverse $f(z)$, and if $f$ is uniformly bounded, then $1=0$ in $A$.

Classically the inverse is always uniformly bounded. Moreover, there is a metatheorem, the fan rule, that allows us to find a bound in concrete cases. Precisely, if the inverse is definable in a 'reasonable' formal system for Bishop-style mathematics, then we can find the bound by a mechanical procedure; see for instance [3, p.394][30]. In the applications below we make an effort to be explicit about the bound. ${ }^{2}$

Proof We have $f\left(z^{\prime}\right)-f(z)=\left(z-z^{\prime}\right) f(z) f\left(z^{\prime}\right)$. Hence $f$ is Lipschitz continuous and differentiable with $f^{\prime}=-f^{2}$. We consider the circle loop $\Gamma_{R}: t \mapsto R e^{2 \pi i t}$ for some $R>|u|$. By Lemma $4, \int_{\Gamma_{R}} f=0$.

On the other hand, for $R$ big enough, $f(z)$ is equal to $\sum \frac{u^{n}}{z^{n+1}}$ over $\Gamma_{R}$. So $\int_{\Gamma_{R}} f$ is equal to $\int_{\Gamma_{R}} d z / z=2 \pi i$. So $1=0$ in $A$.

Let $I$ be an ideal of a Banach algebra $A$. We define a new Banach algebra $A / I$ by taking the new norm to be: $|a|_{I}<r$ if and only if there exists $b$ in $I$ such that $|a-b|<r$. Then $a=0$ in $A / I$ if and only if $a$ belongs to the closure of $I$. By Corollary $1,1=0$ in $A / I$ if and only if 1 belongs to $I$.

We deduce the following method from the previous theorem. To prove that $g$ in $A$ is invertible, it suffices to find $u$ in $A$ such that for all $z$ in $\mathbb{C}, z-u$ is invertible with bounded inverse in $A /\langle g\rangle$.

\section{Application 1: Wiener's Theorem on Fourier series}

We present Wiener's theorem on Fourier series; see for instance [25]. The Banach algebra $B:=l^{1}(\mathbb{Z})$ is the completion of the algebra of sequences in $\mathbb{C}^{\mathbb{Z}}$ of finite support

\footnotetext{
${ }^{2}$ Classically, this theorem implies the Gelfand-Mazur Theorem: a Banach algebra $A$ which is also a field is isomorphic to $\mathbb{C}$. Indeed, from Theorem 2 , for each $u$ in $A$, there exists $\lambda$ such that $u-\lambda$ is not invertible and this implies $u=\lambda$.
} 
with the convolution product $(a * b)_{n}:=\sum a_{i} b_{n-i}$ and the norm $|a|:=\sum\left|a_{n}\right|$. This algebra has the Dirac function $\delta_{0}$ as unit. We write $u$ for $\delta_{1}$. Then $u^{-1}=\delta_{-1}$ and for every $a, a=\sum a_{n} u^{n}$. We see that $B$ is simply an algebra of infinite series under formal multiplication.

For $\lambda$ in the unit circle $\Gamma$, we define $a(\lambda):=\sum a_{n} \lambda^{n}$. Then $|a(\lambda)| \leqslant|a|$.

Theorem 3 Let $f$ in $B$ be such that for all $\lambda$ in $\Gamma,|f(\lambda)| \geqslant \varepsilon$. Then $f$ is invertible.

Proof Let $A:=B /\langle f\rangle$. We show that for all $\lambda$ in $\mathbb{C}, \lambda-u$ is invertible and that the inverse is uniformly bounded. We can then apply Theorem 2 .

If $|\lambda|<1$, then $\left|\lambda u^{-1}\right|=|\lambda| \leqslant r$, for some $r<1$. Hence,

$$
\sum_{n \geq 1}(\lambda / u)^{n}=\left(1-\lambda u^{-1}\right)^{-1}=u(u-\lambda)^{-1} .
$$

The inverse $(u-\lambda)^{-1}$ is bounded by $\left|u^{-1}\right|(1-r)^{-1}=(1-r)^{-1}$.

Similarly, if $|\lambda|>1$, then $|u / \lambda|=|1 / \lambda| \leqslant r$ for some $r<1$. Hence,

$$
\sum_{n \geqslant 1}(u / \lambda)^{n}=(1-(u / \lambda))^{-1}=u^{-1}(\lambda-u)^{-1} .
$$

The inverse $(\lambda-u)^{-1}$ is bounded by $|u|(1-r)^{-1}=(1-r)^{-1}$.

Now consider the case where $\lambda$ is close to the circle. Let $g$ be an element of finite support such that $|f-g|<\varepsilon / 3$. Then

$$
|(g(\lambda)-g)-(f(\lambda)-f)|=|(g-f)(\lambda)+(f-g)| \leqslant 2 \varepsilon / 3 .
$$

Since $f(\lambda)-f=f(\lambda)(\bmod f)$, we have that $g(\lambda)-g$ is invertible $(\bmod f)$. By Lemma 5, we have a uniform bound $M$ on the inverse of $g(\lambda)-g$ in $\Gamma$. Since $g(\lambda)-g$ is a polynomial in $u, \lambda-u$ divides it, say, $(\lambda-u) h=g(\lambda)-g$. So, for all $\lambda$ in $\Gamma, \lambda-u$ is invertible and the inverse is bounded by $M|h|$. Without loss of generality, we assume that $M|h| \geqslant 1$. By Lemma 5, $\lambda-u$ is invertible for all $\lambda$ with ||$\lambda|-1| \leqslant 1 /(2 M|h|)$ and its inverse is bounded by $|M h| /\left(1-\frac{1}{2}\right)=2|M h|$.

Write $\alpha:=1 /(2 M|h|)$. Then either ||$\lambda|-1| \leqslant \alpha$ or ||$\lambda|-1| \geqslant \alpha / 2$. In the latter case, either $|\lambda| \leqslant 1-\frac{\alpha}{2}$ or $|\lambda| \geqslant 1+\frac{\alpha}{2}$. We conclude that $\lambda-u$ is invertible for all $\lambda$ in $\mathbb{C}$ and its inverse is bounded by

$$
\sup \left\{2 M|h|,\left(1-\frac{\alpha}{2}\right)^{-1},\left(1-\left(1+\frac{\alpha}{2}\right)^{-1}\right)^{-1}\right\} .
$$

Being constructive, this reasoning can be seen as an algorithm that computes an inverse of $f$ in $B$. 


\section{Application 2: Wiener's Tauberian Theorem}

Let $C(\mathbb{R})$ be the space of continuous functions of compact support. Let $L:=L^{1}(\mathbb{R})$ be its $L_{1}$-completion. We define $T_{x}: C(\mathbb{R}) \rightarrow C(\mathbb{R})$ by $T_{x}(g)(y):=g(x+y)$. Then $\left|T_{x}(g)-T_{x}(h)\right|=|g-h|$. By extension, we have a continuous function $x \mapsto T_{x}(g), \mathbb{R} \rightarrow$ $L$. For all $g$ in $L$ and $x$ in $\mathbb{R},\left|T_{x}(g)\right|=|g|$. For $f$ in $C(\mathbb{R})$ and $g$ in $L$, we define

$$
f * g:=\int f(x) T_{-x}(g) \mathrm{d} x .
$$

Then $|f * g| \leqslant|f|_{\infty}|g|_{1}$, where $|\cdot|_{\infty}$ denotes the sup-norm. Hence the product $f * g$ can be defined by extension for $f$ and $g$ both in $L$. If both $f, g$ are in $C(\mathbb{R})$, so is $f * g$.

Since

$$
(f * g)(y)=(g * f)(y)=\int f(x) g(y-x) \mathrm{d} x
$$

for $f, g$ in $C(\mathbb{R})$, this holds also for $f, g$ in $L$. So, the algebra $L$ with convolution product is a commutative Banach algebra. (It can be shown that it does not have a unit element.)

Let $g$ be a continuous function with compact support. We define

$$
\hat{g}(p):=\int g(x) e^{-i p x} \mathrm{~d} x .
$$

Let $C_{0}(\mathbb{R})$ denote the set of continuous functions that vanish at infinity. Then $\hat{g}$ in $C_{0}(\mathbb{R})$. This is proved first for $g \in C^{1}(\mathbb{R})$ by integration by parts, and then for general $g \in C(\mathbb{R})$ by a density argument. Moreover, $|\hat{g}(p)| \leqslant|g|$ for all $p$. It follows that we can extend the map $\hat{\cdot}$ and define $\hat{g}$ in $C_{0}(\mathbb{R})$ for $g$ in $L$.

We choose $h$ in $L$ such that $\hat{h}=1$ on $[-M, M]$ and for each $\varepsilon>0$ there exists $\eta$ such that $|\hat{h}| \leqslant 1-\eta$ on $(-\infty,-M-\varepsilon] \cup[M+\varepsilon,-\infty)$. For instance, we can use a de la Vallée Poussin kernel [21].

Theorem 4 If $|\hat{f}|>\varepsilon$ on $[-M, M]$ and $g * h=g$ in $L$, then $f$ divides $g$.

Proof Let $I$ be the ideal $\{k * h-k \mid k \in L\}$, and let $B$ be the Banach algebra $L / I$. Notice that $B$ has $h$ as unit element. We claim that $f$ is invertible in $B$.

The map $\alpha: \mathbb{R} \rightarrow B$ defined by $\alpha(x):=T_{x}(h)$ is continuous. It satisfies $\alpha(0)=h$ and

$$
\alpha(x+y)=T_{x+y} h \stackrel{[h=1]}{=} T_{x+y} h * h=T_{x} h * T_{y} h=\alpha(x) * \alpha(y) .
$$


By Lemma 3 there exists an element $u$ in $B$ such that $T_{x}(h)=e^{u x}$ for all $x$ in $\mathbb{R}$. For all $g$ in $B$,

$$
g=g * h=\int g(x) T_{-x} h \mathrm{~d} x=\int g(x) e^{-u x} \mathrm{~d} x .
$$

We show that $u-\lambda$ is invertible $(\bmod f)$ and with bounded inverse. We can then apply Theorem 2 and deduce that $f$ is invertible in $B$.

We will consider three cases:

(1) $\lambda=i p$ for $|p| \leqslant M$;

(2) $\lambda=i p$ for $|p| \geqslant M+\delta$ and $\delta \geqslant 0$;

(3) $\lambda=r+i p$ and $|r|>\delta$ and $\delta>0$.

By continuity and Lemma 5 these cases are sufficient.

(1) We claim that $i p-u$ is invertible $(\bmod f)$ if $|p| \leqslant M$. Indeed, if we take $N$ such that

$$
\left|\int_{-N}^{N}\right| f\left|-\int\right| f|| \leqslant \frac{\varepsilon}{2(1+|h|)},
$$

then

$$
\begin{aligned}
\left|\int f(x)\left(e^{-i p x}-e^{-u x}\right) \mathrm{d} x-\int_{-N}^{N} f(x)\left(e^{-i p x}-e^{-u x}\right) \mathrm{d} x\right| & = \\
\left|\int_{\mathbb{R} \backslash[-N, N]} f(x)\left(e^{-i p x}-e^{-u x}\right) \mathrm{d} x\right| & \leqslant \\
\int_{\mathbb{R} \backslash[-N, N]}|f|(1+|h|) & \leqslant \frac{\varepsilon}{2} .
\end{aligned}
$$

Since

$$
\int f(x)\left(e^{-i p x}-e^{-u x}\right) \mathrm{d} x=\hat{f}(p)-f,
$$

this integral is invertible $(\bmod f)$; by hypothesis its inverse is bounded by $\frac{1}{\varepsilon}$. By Lemma 5,

$$
\int_{-N}^{N} f(x)\left(e^{-i p x}-e^{-u x}\right) \mathrm{d} x
$$

is invertible $(\bmod f)$. Let $g$ be its inverse. Then $|g| \leqslant \frac{1}{\varepsilon}\left(1-\frac{1}{2}\right)=\frac{2}{\varepsilon}$. Since

$$
e^{-i p x}-e^{-u x}=e^{-i p x}\left(1-e^{(i p-u) x}\right),
$$

this integral is divisible by ip $-u$. It remains to find an explicit bound for the inverse. For all $r, r \mid 1-e^{r x}$ and $\left|\frac{1-e^{r x}}{r}\right| \leqslant 1+e^{|r| x}$, as a simple power series argument shows. 
Consequently, the inverse of $i p-u$ is bounded by $\frac{2}{\epsilon} \int_{-N}^{N}|f(x)|\left(1+e^{r x}\right) \mathrm{d} x$, where $r>|i p-u|$.

(2) We claim that $i p-u$ is invertible with bounded inverse for all $p$ such that $|p| \geqslant M+\delta$. Indeed, if we take $\eta>0$ such that $|\hat{h}(p)| \leqslant 1-\eta$ and $N$ such that

$$
\left|\int_{-N}^{N}\right| h\left|-\int\right| h|| \leqslant \frac{\eta}{2(1+|h|)},
$$

then

$$
\begin{aligned}
\left|\int_{-N}^{N} h(x)\left(e^{-i p x}-e^{-u x}\right) \mathrm{d} x-\int h(x)\left(e^{-i p x}-e^{-u x}\right) \mathrm{d} x\right| & = \\
\left|\int_{\mathbb{R} \backslash[-N, N]} h(x)\left(e^{-i p x}-e^{-u x}\right) \mathrm{d} x\right| & \leqslant \\
\int_{\mathbb{R} \backslash[-N, N]}|h|(1+|h|) & \leqslant \frac{\eta}{2} .
\end{aligned}
$$

Moreover,

$$
\left|\int h(x)\left(e^{-i p x}-e^{-u x}\right) \mathrm{d} x\right|=\hat{h}(p)-h .
$$

The right hand side is invertible since $h$ is the unit of $B$ and $|\hat{h}(p)| \leqslant 1-\eta$. Hence $\int_{-N}^{N} h(x)\left(e^{-i p x}-e^{-u x}\right) \mathrm{d} x$ is invertible with inverse bounded by $\frac{2}{\eta}$. As before, the integral is divisible by $i p-u$ and its inverse is bounded by $\frac{2}{\eta} \int_{-N}^{N}|h(x)|\left|1+e^{r x}\right| \mathrm{d} x$, where $r>|i p-u|$.

We conclude that $i p-u$ is invertible for all $p$ in $\mathbb{R}$ and its inverse is bounded by

$$
d:=\frac{2}{\epsilon} \int(|h(x)|+|f(x)|)\left(1+e^{r x}\right) \mathrm{d} x
$$

where $r>|i p-u|$. By Lemma 5, $r+i p-u$ is invertible for all $p$ in $\mathbb{R}$ and all $r$ such that $|r|<1 / 2 d$. The inverse is bounded by $2 d$.

(3) For $\lambda=r+i p, \lambda-u$ divides

$$
1-e^{(\lambda-u) x}=1-e^{\lambda x} T_{x}(h)=1-e^{-r x} e^{-i p x} T_{x}(h)
$$

and $\left|T_{x}(h)\right|$ is bounded by $|h|$. For $x=\frac{\log (2|h|)}{r}$,

$$
\left|e^{-r x} e^{-i p x} T_{x}(h)\right| \leqslant e^{-r x}|h|=\frac{1}{2} .
$$

So the right hand side of equation (1) is invertible with inverse bounded by $2 e^{-r x}=$ $\frac{2}{2|h|}=\frac{1}{|h|}$. Hence $\lambda-u$ has an inverse with bound $\frac{1}{|h|}$ for $|r|>\varepsilon$. 
We have finished the proof that for each $\lambda, u-\lambda$ is invertible $(\bmod f)$ and of bounded inverse. It follows that we have $k$ such that $f * k=h(\bmod I)$. For $g$ in $L$ such that $g * h=g$, we have $g * I=0$. Hence $f * k * g=g$, and so $f$ divides $g$.

Proposition 1 [21, VI.1.13] Let $g$ in $L$ be such that $\hat{g}$ has compact support. Then there exists $h$ in $L$ such that $g * h=g$. In fact, we can use a de la Vallée Poussin kernel for $h$.

We deduce the following version of Wiener's Tauberian Theorem.

Corollary 2 If $f$ in $L$ is such that $\hat{f}$ never takes the value 0 , then every function $g$ such that $\hat{g}$ is of compact support is in the ideal generated by $f$. Consequently, this ideal is dense in $L$.

Proof Any function in $L$ is the limit of functions in $L$ having their Fourier transform of compact support. We now repeat some facts from Katznelson [21, VI.1.12] whose proofs are elementary, but slightly too long to repeat here. Define the Fejér kernel $K_{\lambda}$ by $K_{\lambda}(x):=\lambda K(\lambda x)$, where $K(x):=\frac{1}{2 \pi}\left(\frac{\sin x / 2}{x / 2}\right)^{2}$. By the inversion formula,

$$
f(x)=\frac{1}{2 \pi} \int \hat{f}(t) e^{i t x} \mathrm{~d} t .
$$

Moreover, $\hat{K}_{\lambda}(t)=\max \left(1-\frac{|t|}{\lambda}, 0\right)$ and one can derive that for $|t| \leqslant \lambda$,

$$
\widehat{K_{\lambda} * f}(t)=\left(1-\frac{|t|}{\lambda}\right) \hat{f}(t)
$$

and equal to 0 otherwise.

Since $f * g=\int g(x) T_{-x} f \mathrm{~d} x$ another way to state this Corollary is the following one.

Corollary 3 Let $f$ be in $L$ such $\hat{f}$ never takes the value 0 . Then the vector space generated by the functions $T_{x} f$ is dense in $L$.

Constructively, the hypothesis that $\hat{f}$ never takes the value 0 should be read as: $\hat{f}$ is bounded away from 0 on any compact set. 


\section{Conclusions and Future work}

To have a point-free description of the spectrum of a Banach algebra was the goal of the work of de Bruijn and van der Meiden [14]. The complexity of this description and the process of finding the proof of the compactness of the spectrum is cited by de Bruijn as one inspiration for his AUTOMATH project $[14,13]$. It would be interesting to have an actual implementation of our work, following already existing work formalising basic analysis [27].

The work of Krivine [23] contains several examples similar to Wiener's results, but in a 'real' framework. One considers respectively $l^{1}(\mathbb{N})$ instead of $l^{1}(\mathbb{Z})$ and $L^{1}\left(\mathbb{R}^{+}\right)$ instead of $L^{1}(\mathbb{R})$. It would be interesting to give a constructive interpretation of these results using the technique presented in $[16,17]$.

Constructive, and choice-free, results on Banach spaces can be interpreted as results on Banach sheaves, or equivalently, Banach bundles [26]. Similarly, constructive results on Banach algebras can be interpreted as results on Banach algebra bundles [22].

\section{Acknowledgements}

We would like to thank the referees for their suggestions which helped to improve the presentation of the paper.

\section{References}

[1] B Banaschewski, C Mulvey, The spectral theory of commutative $C^{*}$-algebras: the constructive Gelfand-Mazur theorem, Quaest. Math. 23 (2000), 465-488.

[2] B Banaschewski, C Mulvey, A globalisation of the Gelfand duality theorem, Ann. Pure Appl. Logic 137 (2006), 62-103; doi:10.1016/j.apal.2005.05.018.

[3] M Beeson, Foundations of Constructive Mathematics, Springer (1985).

[4] E Bishop, D Bridges, Constructive Analysis, Grundlehren der Mathematischen Wissenschaften, Vol 279, Springer (1985).

[5] M Bridger, G Stolzenberg, Uniform calculus and the law of bounded change, Amer. Math. Monthly 106 (1999), 628-635.

[6] D Bridges, Constructive methods in Banach algebra theory, Math. Japon. 52 (2000), 145-161. 
[7] D Bridges, R Havea, Approximations to the numerical range of an element of a Banach algebra, in: From Sets and Types to Topology and Analysis, Oxford Logic Guides 48, Oxford Univ. Press (2005), 293-303; doi:10.1093/acprof:oso/9780198566519.003.0019.

[8] D Bridges, R Havea, Powers of a Hermitian element in a Banach algebra, New Zealand J. Math. 36 (2007), 1-10.

[9] D Bridges, R S Havea, Constructing square roots in a Banach algebra, Sci. Math. Jpn. 70 (2009), 355-366.

[10] D Bridges, R Havea, P Schuster, Finitely generated Banach algebras and local Nullstellensätze, Publ. Math. Debrecen 69 (2006), 171-184.

[11] D Bridges, R Havea, P Schuster, Ideals in constructive Banach algebra theory, J. Complexity 22 (2006), 729-737; doi:10.1016/j.jco.2006.03.004.

[12] D Bridges, R Mines, F Richman, P Schuster, The polydisk Nullstellensatz, Proc. Amer. Math. Soc. 132 (2004), 2133-2140; doi:10.1090/S0002-9939-03-07267-8.

[13] N G de Bruijn, Reflections on Automath, in: Selected papers on Automath, Stud. Logic Found. Math. 133, North-Holland, Amsterdam (1994), 201-228.

[14] N G de Bruijn, W van der Meiden, Notes on Gelfand's theory, Nederl. Akad. Wetensch. Proc. Ser. A 70 = Indag. Math. 29 (1967), 467-474.

[15] P J Cohen, A note on constructive methods in Banach algebras, Proc. Amer. Math. Soc. 12 (1961), 159-163; doi:10.2307/2034144.

[16] T Coquand, About Stone's notion of spectrum, Journal of Pure and Applied Algebra 197 (2005), 141-158; doi:10.1016/j.jpaa.2004.08.024.

[17] T Coquand, B Spitters, Formal topology and constructive mathematics: the Gelfand and Stone-Yosida Representation Theorems, Journal of Universal Computer Science 11 (2005), 1932-1944.

[18] T Coquand, B Spitters, Constructive Gelfand duality for $C^{*}$-algebras, Math. Proc. Camb. Phil. Soc. 147 (2009), 339-344; doi:10.1017/S0305004109002515.

[19] T Coquand, G Stolzenberg, The Wiener lemma and certain of its generalizations, Bull. Amer. Math. Soc. 24 (1991), 1-9; doi:10.1090/S0273-0979-1991-15949-5.

[20] D Hilbert, Über das Unendliche, Math. Ann. 95 (1926), 161-190.

[21] Y Katznelson, An Introduction to Harmonic Analysis, third edition, Cambridge Mathematical Library, Cambridge University Press, Cambridge (2004).

[22] J W Kitchen, D A Robbins, Bundles of Banach algebras, Internat. J. Math. Math. Sci. 17 (1994), 671-680; doi:10.1155/S0161171294000955.

[23] J-L Krivine, Anneaux préordonnés, J. Analyse Math. 12 (1964), 307-326.

[24] H Lombardi, C Quitté, Seminormal rings (following Thierry Coquand), Theoret. Comput. Sci. 392 (2008), 113-127; doi:10.1016/j.tcs.2007.10.007. 
[25] L H Loomis, An Introduction to Abstract Harmonic Analysis, D. Van Nostrand Company, Inc., Toronto-New York-London (1953).

[26] C Mulvey, Banach sheaves, J. Pure Appl. Algebra 17 (1980), 69-83; doi:10.1016/00224049(80)90023-7.

[27] R O'Connor, B Spitters, A computer verified, monadic, functional implementation of the integral, Theoretical Computer Science 411 (2010), 3386-3402; doi:10.1016/j.tcs.2010.05.031.

[28] F Richman, Nontrivial uses of trivial rings, Proc. Amer. Math. Soc. 103 (1988), 1012-1014; doi:10.2307/2047076.

[29] H Takamura, An introduction to the theory of $C^{*}$-algebras in constructive mathematics, in: From Sets and Types to Topology and Analysis, Oxford Logic Guides 48, Oxford Univ. Press (2005), 280-292; doi:10.1093/acprof:oso/9780198566519.003.0018.

[30] A Troelstra, D van Dalen, Constructivism in Mathematics: an Introduction, Volume II, Studies in Logic and the Foundations of Mathematics 123, North-Holland (1988).

[31] N Wiener, Tauberian theorems, Ann. of Math. 33(2) (1932), 1-100.

Thierry Coquand, Computing Science Department, Göteborg University

Bas Spitters, Computer Science Department, Radboud University Nijmegen

Received: 16 March 2010 Revised: 19 November 2010 